\title{
Editorial
}

\section{Advances in Blind Source Separation}

\author{
Andrzej Cichocki ${ }^{1}$ and Frank Ehlers ${ }^{2}$ \\ ${ }^{1}$ Laboratory for Advanced Brain Signal Processing, Brain Science Institute, RIKEN, Hirosawa 2-1, Wako-shi Saitama 351-0198, Japan \\ ${ }^{2}$ NATO Undersea Research Centre, Viale S. Bartolomeo 400, 19138 La Spezia, Italy
}

Received 23 August 2006; Accepted 23 August 2006

Copyright (C) 2007 A. Cichocki and F. Ehlers. This is an open access article distributed under the Creative Commons Attribution License, which permits unrestricted use, distribution, and reproduction in any medium, provided the original work is properly cited.

Blind source separation (BSS) and related topics such as independent component analysis (ICA), sparse component analysis (SCA), or nonnegative matrix factorization (NMF) have become emerging tools in multivariate signal processing and data analysis and are now one of the hottest and emerging areas in signal processing with solid theoretical foundations and many potential applications. In fact, BSS has become a quite important topic of research and development in many areas, especially speech enhancement, biomedical engineering, medical imaging, communication, remote sensing systems, exploration seismology, geophysics, econometrics, data mining, and so forth. The blind source separation techniques principally do not use any training data and do not assume a priori knowledge about parameters of mixing convolutive and filtering systems. Researchers from various fields are interested in different, usually very diverse aspects of BSS. BSS continues to generate a flurry of research interest, resulting in increasing numbers of papers submitted to conferences and journals. Furthermore, there are many workshops and special sessions conducted in major conferences that focus on recent research results. The International Conference on ICA and BSS is a prime example of the attractiveness and research diversity of this field.

The goal of this special issue is to present the latest research in BSS/ICA. We received more than 25 papers of which 10 were accepted for publication. The topics covered in this issue cover a wide range of research areas including BSS theories and algorithms, sparse representations, nonlinear mixing, and some BSS applications.

\section{Theory and Algorithms for ICA/SCA}

In the first paper in this issue, Thomas Melia and Scott Rickard present DESPIRIT algorithm which is an extension of the DUET Blind Source Separation algorithm which can demix an arbitrary number of speech signals using only two anechoic mixtures of the signals. The DUETESPRIT (DESPRIT) Blind Source Separation algorithm extends DUET to situations where sparsely echoic mixtures of an arbitrary number of sources overlap in timefrequency. This paper outlines the development of the DESPRIT method and demonstrates its properties through various experiments conducted on synthetic and real world mixtures.

In the second paper Scott Douglas developed new fixedpoint algorithms for the blind separation of complex-valued mixtures of non-circularly-symmetric, and non-Gaussian independent source signals. Leveraging recently-developed results on the separability of complex-value signal mixtures, he systematically constructed iterative procedures on a kurtosis-based contrast whose evolutionary characteristics are identical to those of the FastICA algorithm of Hyvarinen and Oja in the real-valued mixtures case. The proposed methods inherit the fast convergence properties, computational simplicity, and ease of use of the FastICA algorithm while at the same time extending this class of techniques to complex signal mixtures. For extracting multiple sources, symmetric and asymmetric signal deflation procedures have been employed. Simulation for both noiseless and noisy mixtures indicate that the proposed algorithms have superior finite-sample performance in data-starved scenarios as compared to existing complex ICA methods while performing about as well as the best of these techniques for larger data record lengths.

In the third paper, Fabian J. Theis et al. consider sparse component analysis problem for an overcomplete model using Hough transform. They propose an algorithm which performs a global search for hyperplane clusters within the mixture space by gathering possible hyperplane parameters within a Hough's accumulator tensor. This renders the 
algorithm immune to the many local minima typically exhibited by the corresponding cost function. In contrast to previous approaches, Hough's SCA is linear in the sample number and independent of the source dimension as well as robust against noise and outliers. Experiments demonstrate the flexibility of the proposed algorithm.

\section{Blind Deconvolution: Models and algorithms}

Bin Xia and Liqing Zhang introduced a cascade demixing structure for multichannel blind deconvolution in nonminimum phase systems. To simplify the learning process, they proposed to decompose the demixing model into a causal finite impulse response (FIR) filter and an anticausal scalar FIR filter. A permutable cascade structure is constructed by two subfilters. After discussing the geometrical structure of FIR filter manifold, they proposed to use the natural gradient algorithms for both FIR subfilters. Furthermore, they derived the stability conditions of algorithms using the permutable characteristic of the cascade structure. Finally, computer simulations are provided to show good learning performance of the proposed method.

Stefan Winter et al. addressed the problem of underdetermined BSS. While most previous approaches are designed for instantaneous mixtures, they proposed a time-frequency domain algorithm for convolutive mixtures. Starting from a general maximum a posteriori (MAP) approach, they proposed a two-step approach. In the first step they estimated the mixing matrix based on hierarchical clustering, assuming that the source signals are sufficiently sparse. The assumption of Laplacian priors for the source signals leads in the second step to an algorithm for estimating the source signals. It involves L1-norm minimization of complex numbers due to the time-frequency-domain approach. They compared a combinatorial approach initially designed for real numbers with a second-order cone programming (SOCP) approach for complex numbers. The advantage of the proposed algorithm is lower computational cost for problems with low input/output dimensions.

Robert Aichner et al. proposed an algorithm combining advantages of broadband algorithms with the computational efficiency of narrowband techniques. By selective application of the Szego theorem which relates properties of Toeplitz and circulant matrices, normalization is derived as a special case of the generic broadband algorithm. This results in a computationally efficient and fast converging algorithm without introducing typical narrowband problems such as the internal permutation problem or circularity effects. Moreover, a regularization method for the generic broadband algorithm is presented and subsequently also derived for the proposed algorithm. Experimental results in realistic acoustic environments show improved performance of the novel algorithm compared to previous approximations.

Ricardo Suyama et al. proposed a method for source separation of convolutive mixture based on nonlinear prediction error filters. This approach converts the original problem into an instantaneous mixture problem, which can be solved by any of the several existing methods in the literature. They employed fuzzy-filters to implement the prediction-error filter, and the efficacy of the proposed method is illustrated by some examples.

\section{Nonlinear ICA}

Thang Viet Nguyen and Jagdish Chandra Patra proposed a geometric approach for nonlinear independent component analysis (ICA). Nonlinear environment is modeled by the standard post nonlinear (PNL) scheme. To eliminate the nonlinearity in the observed signals, a novel linearizing method named as geometric post nonlinear ICA (gpICA) is introduced. Thereafter, a basic linear ICA is applied on these linearized signals to estimate the unknown sources. The proposed method is motivated by the fact that in a multidimensional space, a nonlinear mixture is represented by a nonlinear surface while a linear mixture is represented by a plane, a special form of the surface. Therefore, by geometrically transforming the surface representing a nonlinear mixture into a plane, the mixture can be linearized. Through simulations on different data sets, superior performance of gpICA algorithm has been shown with respect to other algorithms.

\section{Applications}

Iván Durán-Díaz and Sergio A. Cruces-Alvarez addressed the important problem of the blind detection of a desired user in an asynchronous DS-CDMA communications system with multipath propagation channels. Starting from the inverse filter criterion introduced by Tugnait and $\mathrm{Li}$, they propose to tackle the problem in the context of the blind signal extraction methods for ICA. In order to improve the performance of the detector, they presented a criterion based on the joint optimization of several higher-order statistics of the outputs. An algorithm that optimizes the proposed criterion is described, and its improved performance and robustness with respect to the near-far problem are corroborated through simulations. Additionally, a simulation using measurements on real software-radio platform at $5 \mathrm{GHz}$ has also been performed.

Finally, Loukianos Spyrou et al. presented application of BSS to separation and localization of P300 sources and their constituent subcomponents for both visual and audio stimulations for EEG signals. An effective constrained blind source separation (CBSS) algorithm is developed for this purpose. The algorithm is an extension of the Infomax BSS system for which a measure of distance between a measured P300 and the estimated sources is used as a constraint. During separation, the proposed CBSS method attempts to extract the corresponding P300 signals. The locations of the corresponding sources are then estimated with some indeterminacy in the results. It can be seen that the locations of the sources change for a schizophrenic patient. The experimental results verify the statistical significance of the method and its potential application in the diagnosis and monitoring of schizophrenia. 


\section{ACKNOWLEDGMENTS}

The guest editors of this special issue are much indebted to their authors and reviewers, who put a tremendous amount of effort and dedication to make this issue a reality.

Andrzej Cichocki Frank Ehlers

Andrzej Cichocki was born in Poland. He received the M.S. (with honors), Ph.D. and Habilitate Doctorate (Dr.Sc.) degrees, all in electrical engineering, from the Warsaw University of Technology (Poland) in 1972 , 1975, and 1982, respectively. He is the coauthor of three international and successful books (two of them translated to Chinese): Adaptive Blind Signal and Image Processing (John Wiley, 2002) MOS Switched-

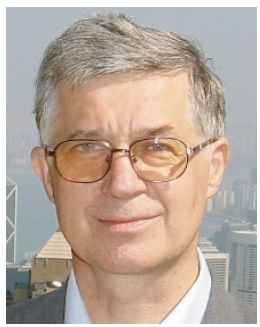
Capacitor and Continuous-Time Integrated Circuits and Systems, (Springer-Verlag, 1989) and Neural Networks for Optimization and Signal Processing (J. Wiley and Teubner Verlag, 1993/1994) and author or coauthor of more than three hundreds papers. He is Editor-in-Chief of Journal of Computational Intelligence and Neuroscience and Associate Editor of IEEE Transactions on Neural Networks. Since 1997 he is the head of the laboratory for Advanced Brain Signal Processing in the Riken Brain Science Institute, Japan.

Frank Ehlers obtained the Diploma degree in physics from the Christian-AlbrechtsUniversity Kiel, Germany, in 1995; he did the work for his thesis on "Linear convolutive blind source separation" in the group of Professor Schuster. He obtained the Dr.rer.nat. degree in theoretical physics, in 1998 from the Christian-Albrechts-University Kiel, Germany. He wrote the Ph.D. thesis on "Non-linear blind source separa-

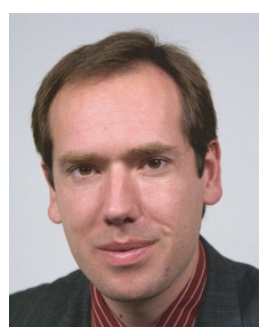
tion" under the supervision of Prof. Schuster. Since March 1998, he has been working at the Federal Armed Forces Underwater Acoustics and Geophysics Research Institute (FWG) in Kiel, Germany, where he focused on signal processing for detection, tracking, classification, sensor control, and fusion. Since April 2006, he is working as a Programme Manager for "Multisensor systems and methods" at the NATO Undersea Research Centre, La Spezia, Italy. He actively conducts both application oriented research as well as more fundamental research in the broader fields of data fusion and collaborative signal processing. He has served and serves as a member on a number of different international programming committees for conferences such as EUSIPCO and FUSION. He is also a member of the Editorial Board of the EURASIP Journal on Applied Signal Processing and performs reviewing activities for different scientific journals. 